\title{
Combining Image Processing Techniques and Mobile Sensor Information for Marker-less Augmented Reality Based Reconstruction
}

\author{
I. S. Weerakkody \\ School of Computing, University of \\ Colombo \\ Colombo, Sri Lanka \\ Email: weerakkody.shehan [AT] \\ gmail.com
}

\author{
K. D. Sandaruwan \\ School of Computing, University of \\ Colombo \\ Colombo, Sri Lanka \\ Email: dsr [AT] ucsc.cmb.ac.lk
}

\author{
N. D. Kodikara \\ School of Computing, University of \\ Colombo \\ Colombo, Sri Lanka \\ Email: $n d k$ [AT] ucsc.cmb.ac.lk
}

Abstract - Marker-less Augmented Reality(AR) based recon- struction using mobile devices, is a near impossible task. When considering vision based tracking approaches, it is due to the lack of processing power in mobile devices and when considering mobile sensor based tracking approaches, it is due to the lack of accuracy in mobile Global Positioning System(GPS).

In order to address this problem this research presents a novel approach which combines image processing techniques and mobile sensor information which can be used to perform precise position localization in order to perform augmented reality based reconstruction using mobile devices. The core of this proposed methodology is tightly bound with the image processing technique which is used to identify the object scale in a given image, which is taken from the user's mobile device. Use of mobile sensor information was to classify the most optimal locations for a given particular user location.

This proposed methodology has been evaluated against the results obtained using $10 \mathrm{~cm}$ accurate Real-Time Kinematic(RTK) device and against the results obtained using only the Assisted Global Positioning System(AGPS) chips in mobile devices. Though this proposed methodology require more processing time than A-GPS chips, the accuracy level of this proposed methodology outperforms that of A-GPS chips and the results of the experiments carried out further convince that this proposed methodology facilitates improving the accuracy of position local- ization for augmented reality based reconstruction using mobile devices under certain limitations.

Keywords- augmented reality based reconstruction, position localization, image processing, mobile sensor information

\section{INTRODUCTION}

Augmented reality is the integration of digital information with the user's environment in real time [1]. Unlike virtual reality, which creates a totally virtual environment, augmented reality uses the existing environment and overlays new digital information on top of it. This information is mostly animations or contextual digital information [1]. In augmented reality digital information is added to the real environment through some devices (goggles, smartphones, Head Mounted Displays) and the information presented often requires the actual precise location of the user. For instance, when visiting some ruins, one could see a depiction of what the site used to look like superimposed over the remains. This is useful not only for historical representations but also for educational purposes(architects, engineers) [2].

Popularity over augmented reality has rapidly increased during the past decade. Some consider augmented reality as one of the most revolutionary inventions in recent years [3]. One of the reasons for its growing popularity is, its unique ability to provide a mediated perception of the real- world environment by seamlessly integrating it with computer- generated content [4].

This research mainly concerns augmented reality based reconstruction for relatively large objects such as partially destroyed ancient monuments. With this proposed solution users would have the opportunity to see ancient monuments in their full structure at their original location. Hence, users will have the chance to relive the glory and beauty of these ancient monuments. When considering augmented reality based reconstruction for such large objects, aspects such as position accuracy, stability and hardware support are important facts [5].

Even though at present there are many ongoing researches and studies on augmented reality, precise augmentation with mobile devices using marker-less approach has been a constant issue [1]. Typically in augmented reality, cameras and other sensors are used to track user's absolute location so that when the user moves, the object stays in the programmed position [6]. But as mobile devices have a relatively low processing power and as they are equipped with relatively low cost GPS chips [7] and as GPS localization requires the line of 
site to four or more satellites which makes it even harder to accurately perform position localization in some urban environments [8], doing precise augmentation using the maker-less approach for mobile devices has become an even bigger issue in recent past.

Even though the emerging 5G communication technologies act as critical enablers for future mobile augmented reality applications to achieve ultra-low latency and extremely high data rates while Multi-access Edge Computing (MEC) brings enhanced computational power closer to the users to complement mobile augmented reality [9], not all mobile devices are capable of handling 5G communication technology. Our work is more concentrated on any mobile device that is equipped with a camera and a GPS chip.

In this research it is expected to combine the two approaches of mobile sensor information based augmented reality and vision based tracking for outdoor augmented reality to overcome the disadvantages of using a single approach. Furthermore efficiency and the accuracy of the augmented reality based reconstruction has been increased using the implemented method for position localization using mobile devices which uses both image processing techniques and mobile sensor information.

\section{RELATED WORK}

\section{A. Augmented reality using sensor information}

According to a research done on global pose estimation using multi sensor fusion, outdoor augmented reality typically requires tracking in unprepared environments [10]. For global registration, GPS is currently the best sensing technology, but its precision and update rate are not sufficient for high quality tracking. In this research the researchers have presented a system that uses Kalman filtering for fusion of Differential Global Positioning System(DGPS) or RTK based GPS with barometric heights and also an inertial measurement unit with gyroscopes, magnetometers and accelerometers to improve the transient oscillation. They have developed a hardware tracking module using Differential-GPS or Real-Time Kinematic based GPS. This hardware tracking module is suitable to use with handheld augmented reality devices due to its small weight and form factor [10]. A typical DGPS receiver is accurate for less than one meter [11]. Hence, with the hardware module these researchers were able to obtain higher accuracy rate for position localization.

There have been hybrid approaches which combined the data of the compass, accelerometer and the GPS together in order to calculate where the object should be augmented in the field of view without any actual processing of the real image [10]. With the compass it is possible to tell the direction which the device is pointing at and the accelerometer is used to calculate orientation of the device using gravitation to its advantage [12]. But typically, inertial sensors are subjected to drift and magnetometer measurements are distorted by electromagnetic fields in the environment.

\section{B. Marker-based augmented reality using Vision based track- ing}

Fiducial markers can be introduced and localized in the environment, so that online localization can be achieved by simply recognizing them using an appropriate sensing pipeline [1]. Fiducial markers typically consists of small colored LEDs (Light Emitting Diode) or pieces of paper. This tracking method may lead to more accurate localization results than the maker less approaches [1]. But it requires intrusive and accurate positioning of markers within the environment and markers could clutter the physical environment as well [1]. And also there is a certain possibility that these markers could be fully or partially destroyed with the time due to environmental effects.

C. Marker-less augmented reality using Vision based tracking As the computational power of devices used for augmented reality applications improved, it became possible to register the pose of the camera in real time using features which already exist in the natural environment [1]. Complicated image processing algorithms are used to detect features in the captured images which are unique in their surroundings, such as points, corners and the intersections of lines. In a research done in 2014, for each of these features an unique "descriptor" is calculated which allows for identification and differentiation of each feature [1]. By matching features detected in the scene with those detected in the object to be tracked, the pose can be computed using similar algorithms as those used in the Fiducial marker techniques [1]. Some of the more common natural feature detection and description algorithms include Scale Invariant Feature Transform(SIFT), Speeded Up Ro- bust Features(SURF), Binary Robust Independent Elementary Feature(BRIEF), Oriented FAST and Rotated BRIRF(ORB), Binary Robust Invariant Scalable Keypoints(BRISK) and Fast Retina Keypoint(FREAK) [13]. When considering vision based tracking for outdoor augmented reality, performance is a critical issue. To increase the accuracy level of a vision based tracking system the number of reference images should be increased. When increasing the number of reference images, then the number of comparisons will increase as well [5]. Hence, the tracking time would increase rapidly. Since mobile phones are not equipped with high computational power (with respect to laptops and other devices used for augmented reality) this is a major problem that needs to be solved. Also when considering an outdoor environment lighting condition is also a fact to be concerned with [5].

\section{Location positioning for an indoor environment}

In a research done in 2010 by Gerhard Reitmayr, et al. the researchers have presented a vision-based location positioning system using augmented reality technique for indoor naviga- tion [14]. The method proposed in this paper automatically recognizes a location using image sequences of that indoor environment, and it realizes augmented reality by seamlessly overlaying the users view with location 
information. In or- der to obtain these location positions in this research, the researchers have used a preconstructed image database and location models, which consists of locations and paths between locations, of an indoor environment. Location is recognized by using prior knowledge about the layout of the indoor environment. To carry out the experiments of this research the researchers have used highly sophisticated equipment such as wireless camera, mobile personal computer has been used together with a Head Mounted Display. In this research the researcher have not used GPS-based location positioning systems to a great extent as GPS radio signals have difficulty in penetrating building walls. Since this proposed approach is for indoor usage and indoor environments usually consists of high number of walls the accuracy level given by a GPS-based location positioning systems will be relatively low. Results of the experiments carried out in this research have shown that this proposed system produces an average location recognition success rate of $89 \%$ in an indoor environment.

\section{RESEARCH DESIGN AND IMPLEMENTATION}

The methodology proposed in this paper consists several phases which are briefed in the next few sections. Some of these phases are a onetime process for certain site (particularly the first two phases) and some of the phases needs to be carried out repeatedly for each user that visits the site.

\section{A. Obtaining reference images}

Initially a set of locations were selected from the site that was going to be reconstructed. Named those set of locations as reference locations(for explanation purposes) and calculated their accurate GPS coordinates using an $10 \mathrm{~cm}$ accurate RTK device. Each of these selected locations were a predefined distance away from the object. Each of these selected reference locations were associated with a bearing value which is the angle of which, someone would look at the object from that particular location. From each of these selected locations an image was captured. This image was zoomed into the object so that more than $90 \%$ of the image is the object itself. These images were named as reference images which will be used as template images when performing template matching. Figure 1 further illustrates how those set of locations were selected. When implementing this phase of the research design a set of 32 locations were selected and each of the locations were

$5 \mathrm{~m}$ away from the object. Number of reference locations and the distance to the object from each reference location is highly dependent on the scale of the object (6ft x $6 \mathrm{ft} \times 6 \mathrm{ft}$ object was used during the experiments).

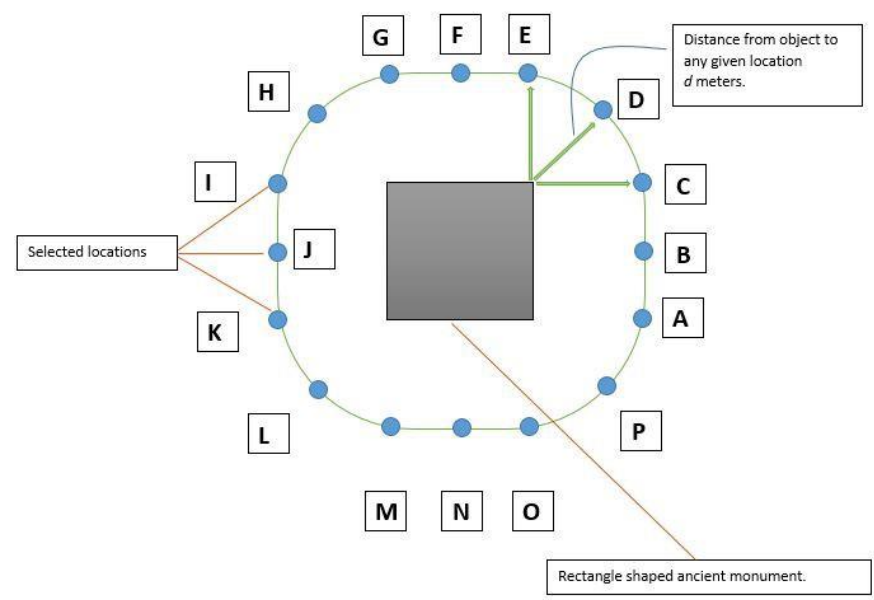

Figure 1. Obtaining reference locations.

\section{B. Deriving functions for each reference location}

Once the reference images are obtained the next phase of the research design would be to derive functions for each of the selected reference locations. These functions represent the scale deviation of the object in the image against the distance to the object from the location where that image was captured by the user. These functions will be useful when determining the distance from the user's location to the object when the scale of the object in the user image is known. These derived function will have the distance to the object from a particular location as the domain and the scale of the object in an image as the co-domain.

When deriving these functions a set of three images were captured $5 \mathrm{~m}, 6 \mathrm{~m}$ and $7 \mathrm{~m}$ away from the object respectively, for each of the selected reference locations. Increasing this number of three(images per reference location) would increase the accuracy of the derived functions. Once the set of three images were captured, a newly implemented algorithm was used to identify the scale of the object in each of the captured images. This data was then used together with linear regression with least square estimation technique [15] to derive the functions for each of the reference locations.

\section{Identifying the most optimal set of reference locations}

Once the users arrives to the site with their mobile phones, it is assumed that a user captures a geo tagged image of the site without zooming in or out. It is also assumed that this image has a full portion of the object as well. Comparing the user image with all the captured reference images won't be the most optimal method since efficiency is one of the factors that has to be considered in this type of an augmented reality based reconstruction. Hence, the closest eight reference locations were selected based on the user's approximate location which is produced by the geo tag of the user image which the user captures. When calculating the closes eight locations great circle distance finding method with Vincenty equation [16] was used. 
D. Using template matching to find the best matching refer-ence location

Reducing the selected eight optimal reference images to one particular reference image that best matches with the user's location is done through the implemented algorithm. This implemented new algorithm has seen the usage of ex-isting typical template matching algorithm. Drastic changes in day time lighting conditions would have a negative effect on this implemented template matching algorithm. Before approaching with this implemented algorithm, the scale of the image taken by the user was reduced to $20 \%$ of it's original scale in order to improve the performance of the implemented algorithm. This scale value is highly dependent on the scale of the object which was used for the evaluation and it may be a different value for an object with a different scale. Even though this implemented algorithm has provided substantially accurate results throughout the evaluation process and has been optimized in certain ways, this approach is similar to a typical brute force mechanism. Hence, there can be future enhancements to optimize this algorithms in order to improve its efficiency.

The implemented approach of reducing the set of eight optimal reference location in order to find the best matching reference locations based on the user's approximate location contains the following steps.

- Obtain the user image and set it as the source image.

- Scale down the source image to a predefined value in order to reduce the number of pixels in the user image and improve the performance of this algorithm,

- Obtain the most optimal set of reference images based on the users current geo location.

- Scale down all the optimal reference images to a certain value (this value depends on the scale of the object) and set them as template images for the initial iteration.

- Perform typical template matching algorithm using user image and each of the scaled down template images and compute a correlation score.

- Increase the scale of the template images minimally and use them as template images for the next iteration and perform template matching.

- Until the scale of template image reaches a predefined value (this value depends on the scale of the object) repeat the previous two steps.

- Obtain the highest 10 correlation scores of each template and select the template which has the largest sum over its 10 highest correlation scores and set it as the best matching template.

Algorithm 1 is the pseudo code of the implemented algorithm. This was also used to identify the scale of the object in the user image captured by the user.

\section{E. Obtaining the scale of the object in the user image}

Result of the previous phase provide us with the best matching template image and the reference location of which that template image was captured. More than $90 \%$ of all the template images (Reference images) are filled with the object itself. Hence, the scale of the template image when it best matches with the source image can be assumed as the scale of the object in the user image.

When implementing this phase top ten correlation values of the best matching template were obtained. Average scale values of the best matching template which had the highest ten correlation scores was taken and assumed as the scale of the object in the user image.

\section{F. Obtaining users accurate location}

As the scale value of the user image has been identified and the best matching location based on the user location is known, obtain the function at the best matching location and solve that particular function using the scale of the object in the user image in order to obtain the distance from the user's location to the object.

Reference locations were selected in a way that each of those locations are a predefined distance away from the object. Best matching location is also one of those reference locations. Hence the distance from the object to the best matching location is known.

As the distance from the user to the object is known and also the distance from the best matching location to the object is also known, in order to obtain the distance from the user to the best matching location, subtract distance from the best matching location to the object from the distance to the object from the user.

As the accurate GPS coordinates (10cm accurate) of the best matching location is known together with the bearing value associated with the best matching location, use great circle distance finding method with Vincenty equation [16] together with the distance from the user to the best matching location in order to obtain user's accurate GPS coordinate 
SourceImage = user image;

GeoSource $=$ Geo tag of the source image;

OptimalImageArray[] = optimal reference images ;

SourceImage $=$ Scale $($ SourceImage $) * 0.2(20 \%)$;

BestMatchRate $=0$;

BestMatchLocation =

0 ; BestMatchScale = 0;

StartingScale; FinalScale; StepSize;

for TImage in OptimalImageArray[] do

TemplateImage $=$ TImage , AccuracyArray $=[]$

for $\mathrm{j}$ in range(StartingScale, FinalScale, 1)

do

Template $=$

Scale(TemplateImage)*j*StepSize; Perform

template matching (SourceImage, Template)

TMCCOEFFNORMED;

Accuracy=Matching Accuracy;

AccuracyArray.append(j,Accuracy);

end

SortedAccuracyArray[] = Sort (AccuracyArray on

Accuracy);

MatchRate $=$ average of first 10 Accuracy in

SortedAccuracyArray[];

MatchScale $=$ average of first $10 \mathrm{j}$ values in

SortedAccuracyArray[];

if BestMatchRate; MatchRate then

BestMatchRate $=$ MatchRate;

BestMatchLocation = location of Image;

BestMatchScale = MatchScale;

end

Algorithm 1: Pseudo code of the implemented algorithm

\section{G. Augmentation}

- User's accurate GPS coordinates are now obtained as a result of the previous phases.

- Geo location of the partially destroyed object is known as pre-knowledge.

- Hence, user's accurate location relative to the object can be calculated.

- Using this relative position initial augmentation can proceed.

- To keep track of the augmented three dimensional object, use extended tracking mechanism.

H. High level view of the proposed methodology

Figure 2 depicts a high level view of the process that is carried out in the proposed methodology in order to perform the necessary augmented reality based reconstruction using mobile devices.

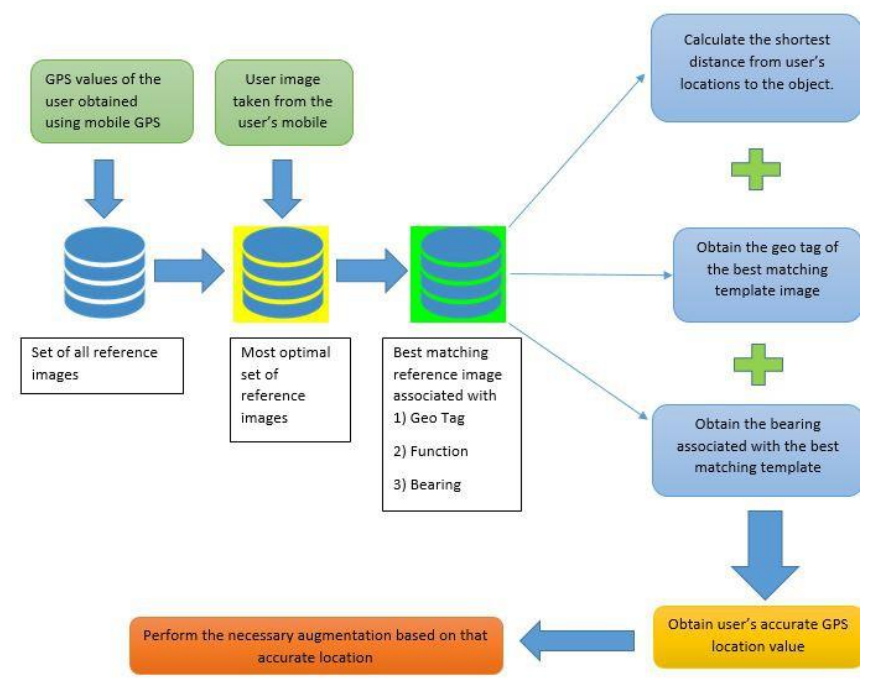

Figure 2. Research design overview.

\section{EVALUATION AND RESULTS}

The main problem that arises when considering augmented reality based reconstruction using mobile devices, is the low accuracy of the position localization achieved by using the A-GPS chips in the mobile device. Purpose of this research paper is to bridge that gap and provide an accurate and efficient position localization method which can be used with mobile devices for augmented reality based reconstruction. In order to evaluate the proposed methodology the same data set was used to conduct three different experiments. Below are the brief explanations of those three experiments.

1) Experiment 1 - Template matching using original images with image size increasing by $0.01 \%$ in each iteration (step size $0.01 \%$ ).

2) Experiment 2 - Template matching using original images with image size increasing by $0.1 \%$ in each iteration (step size $0.1 \%$ ).

3) Experiment 3 - Template matching using gray scaled images with image size increasing by $0.01 \%$ in each iteration (step size $0.01 \%$ ).

When evaluating this proposed methodology a set of 26 locations were used and the GPS coordinates of those 26 location given by a typical A-GPS(Assisted-GPS) chip in a mobile device was recorded and those data were compared against the GPS coordinates of those 26 locations given by a $10 \mathrm{~cm}$ accurate RTK device. In the next phase for all the three experiments GPS coordinates of the 26 locations given by the proposed methodology were compared against the GPS coordinates of the 26 locations given by a $10 \mathrm{~cm}$ accurate RTK 


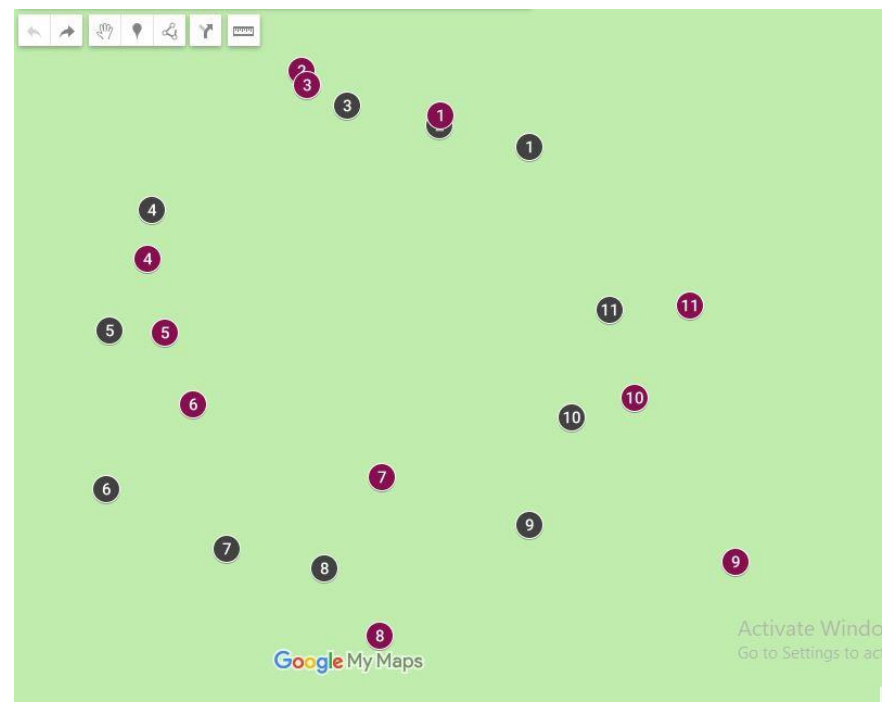

Figure 3. Mobile A-GPS data against RTK data.

Figure 4 is a Google map image of 11 of the above recorded position localization points obtained using $10 \mathrm{~cm}$ accurate RTK device(gray points) and position localization points obtained using the methodology proposed in this research study(blue markers).

Table 1 depicts a summary of all the position localization data obtained throughout the evaluation process using the pro- posed methodology and by using the existing GPS localization method in mobile devices which uses AGPS.

Table 1: Summary of Accuracy Levels

$\begin{array}{lcccccc}\text { Error(cm) } & 0-10 & 10-40 & 40-150 & 150-500 & 500-800 \\ \text { A-GPS } & 0.0 \% & 0.0 \% & 7.69 \% & 73.08 \% & 19.23 \% \\ \text { Exp 1 } & 30.77 \% & 46.15 \% & 23.08 \% & 0.0 \% & 0.0 \% \\ \text { Exp 2 } & 34.62 \% & 26.92 \% & 26.92 \% & 11.54 \% & 0.0 \% \\ \text { Exp 3 } & 30.77 \% & 42.31 \% & 23.08 \% & 3.85 \% & 0.0 \% \\ \text { Avg of all Exp 32.05\% } & 38.46 \% & 24.36 \% & 5.13 \% & 0.0 \%\end{array}$

Table 2 depicts a summary of all the feedback time data obtained when using the proposed methodology for localization for all the three carried out experiments.

Table 2: Summary of Efficiency of the Proposed Methodology

$\begin{array}{cccc} & \text { Experiment } 1 & \text { Experiment } 2 & \text { Experiment } 3 \\ \text { Feedback time } & 828.75 \mathrm{~s} & 101.55 \mathrm{~s} & 601.84 \mathrm{~s}\end{array}$

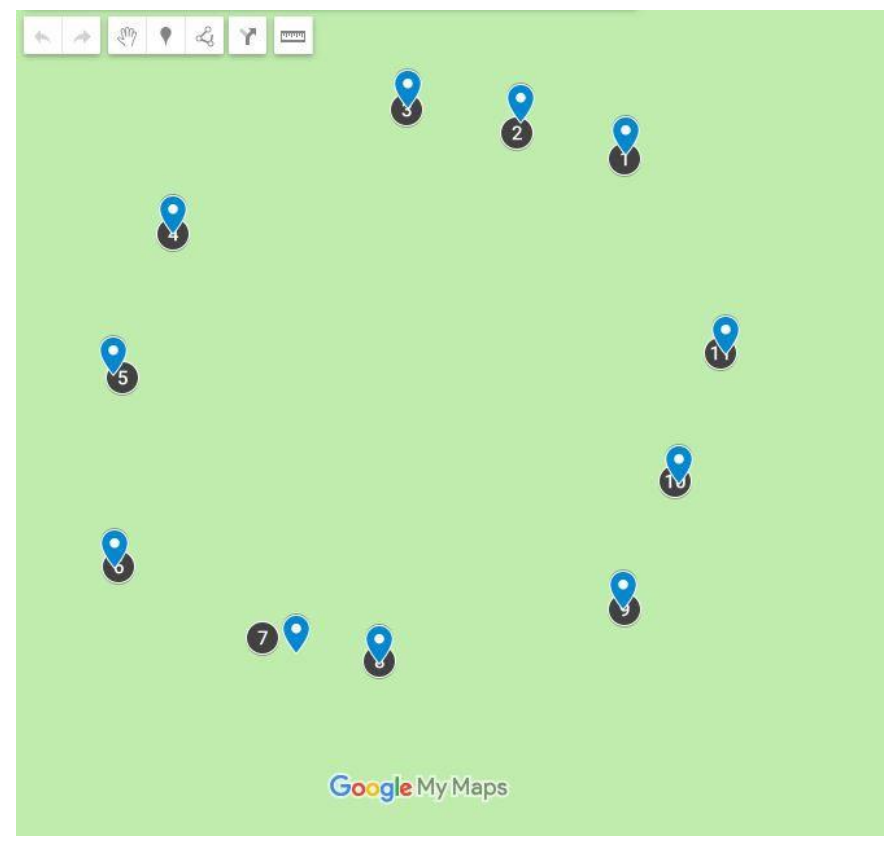

Figure 4. Mobile A-GPS data against RTK data.

Average processing time obtained in each of the above three experiments were 828.75s, 101.55s and $601.84 \mathrm{~s}$ respectively. Even though the accuracy of the proposed methodology is not dependent on the hardware equipment used for the evaluation the average processing time is highly dependent on the low-end server machines used throughout the evaluation. Even with this low-end server machine, with the parameters used in experiment 2, the proposed methodology has been able to provide a feedback by requiring only 101 seconds. This amount of time is minimal compared to the feedback times obtained in other two experiments. Even this value can be optimized to be even less with the use of a high-end server machine.

\section{CONCLUSION}

Even though the methods used in Experiment 1 and Experiment 3 did not provide a timely feedback, method used in Experiment 2 provided a feedback consuming a minimal amount of time compared to the other two experiments. Considering the accuracy level of the localization value, this proposed methodology got some promising results which were substan- tially better than the existing position localization methodol- ogy of mobile devices. Out of the 26 evaluated locations, posi- tion localization values using mobile devices did not have any location matched with at least $40 \mathrm{~cm}$ accuracy level whereas when using the proposed methodology, an average of $70.51 \%$ of the evaluated locations were matched with a accuracy level of $40 \mathrm{~cm}$ or better. Out of the 26 evaluated locations, $92.31 \%$ were not matched with at least $150 \mathrm{~cm}$ accuracy level when using A-GPS chips in 
mobile devices to perform the position localization. But when considering the proposed methodology only an average of $5.13 \%$ of the evaluated locations were not matched with at least $150 \mathrm{~cm}$ accuracy. The definitive goal of this research project was to combine image processing techniques and mobile sensor information in order to achieve less time consuming and accurate position localization using mobile devices for augmented reality based reconstructions of large objects. With the results of Experiment 1, Experiment 2 and Experiment 3 it can be said that the primary goal of this research was achieved. Considering the secondary goals and objectives, the implemented algorithm in section III was the algorithm which was used to identify the scale of an object in the user image and it was further developed and trained using linear regression to accurately calculate users position from a given new user image and the proposed methodology was successfully evaluated against the assumed ground truth data which was obtained using a RTK device. Hence, it can be said that secondary goals and objectives has been achieved under certain conditions. Therefore the proposed methodology will be useful for position localization for augmented reality based reconstructions using mobile devices.

\section{DISCUSSION AND FUTURE WORK}

Improving this proposed methodology so that the negative effect of drastic changes in the day time lighting condition is reduced. This would be a much needed future enhancement in order to provide the user with the experience of AR reconstruction with a high accuracy level during the day time. Efficiency wise, proposed approach in this paper requires relatively high amount of time to provide a feedback. Even though this is down to the lowend devices used throughout the evaluation, this feedback time could also be reduce by optimizing the implemented algorithm. When augmenting the necessary object occlusion handling was not considers. Hence, one other future implication would be to handle occlusion while augmenting the object and provide the user with much more realistic AR experience.

AR is growing rapidly and its applications are increasing everyday. Most of this rapid growth is down to the improvement of smart mobile devices. But when considering augmented reality based reconstruction, mobile devices are not equipped with necessary sensors and the processing power to handle this task.

In 2018 May, Google released there new ARCore software which is capable of many AR applications including augmented reality based reconstruction using mobile devices. Although this software has the capability to handle AR based reconstruction with mobile devices, most of the mobile devices are not capable of handling this software. That is largely due to the unique sensors that this software requires which are not necessarily built into every smart mobile device. device. Throughout this research as the RTK device has a relatively higher accuracy $(10 \mathrm{~cm})$ compared to a mobile device, data given by the RTK device were taken in as the ground truth.

Figure 3 is a Google map image of 11 of the above recorded position localization points obtained using $10 \mathrm{~cm}$ accurate RTK device(gray points) and position localization points obtained using A-GPS chips in mobile devices(red points).

\section{ACKNOWLEDGMENT}

A special thanks goes out to Centre for Research and Development, Ministry of Defense, Sri Lanka for providing the necessary RTK devices

\section{REFERENCES}

[1] Mark Billinghurst, Adrian Clark, Gun Lee. (2014). A Survey of Aug- mented Reality (Vol. 8). Foundations and Trends in Human-Computer Interaction. doi:10.1561/1100000049 [2] Slater Mel, Gonzalez-Liencres Cristina, Haggard Patrick, Vinkers Char- lotte, Gregory-Clarke Rebecca, Jelley Steve, Watson Zillah, Breen Graham, Schwarz Raz, Steptoe William, Szostak Dalila, Halan Shiv- ashankar, Fox Deborah, Silver Jeremy. (2020). The Ethics of Realism in Virtual and Augmented Reality. Frontiers in Virtual Reality, vol. 1 (2020). https://doi.org/10.3389/frvir.2020.00001

[3] He, Z., Wu, L., Li, X. (2018). When are meets tech: The role of aug- mented reality in enhancing musuem experinces and purchase intentions. Tourism MANAGEMENT, 68, 127139.

[4] Han, D. I. D., tom Dieck, M. C., Jung, T. (2019). Augmented Reality Smart Glasses (ARSG) visitoradoption in cultural tourism. Leisure Studies, 116.

[5] Didier Stricker, Thomas Kettenbach. (2001). Real-time and Marker- less VisionBased Tracking for Outdoor Augmented Reality Applica- tions. IEEE and ACM International Symposium on Augmented Reality (ISAR01)

[6] Miller MR, Jun H, Herrera F, Yu Villa J, Welch G, Bailenson JN (2019) Social interaction in augmented reality.PLoS ONE 14(5): e0216290. https://doi.org/10.1371/journal.pone.0216290

[7] Paul A. Zandbergen, Sean J. Barbeau. (2011, JULY). Positional Accu- racy of Assisted GPS Data from High-Sensitivity GPS-enabled Mobile Phones. THE JOURNAL OF NAVIGATION, 64, 381399.

[8] Du, H., Zhang, C., Ye, Q. et al. A hybrid outdoor localization scheme with high-position accuracy and low-power consumption. J Wireless Com Network 2018, 4 (2018). https://doi.org/10.1186/s13638-017-1010-

4

[9] Y. Siriwardhana, P. Porambage, M. Liyanage and M. Ylianttila, "A Survey on Mobile Augmented Reality With 5G Mobile Edge Com- puting: Architectures, Applications, and Technical Aspects," in IEEE Communications Surveys and Tutorials, vol. 23, no. 2, pp. 1160-1192, Secondquarter 2021, doi: 10.1109/COMST.2021.3061981.

[10] Gerhard Schall, Daniel Wagner, Gerhard Reitmayr, Elise Taichmann, Manfred Wieser, Dieter Schmalstieg, Bernhard Hofmann-Wellenhof. (2009). Global Pose Estimation using MultiSensor Fusion for Outdoor Augmented Reality. IEEE International Symposium on Mixed and Augmented Reality 2009, (pp. 153- 162). Orlando, Florida, USA.

[11] Vassilios Vlahakis, John Karigiannis, Manolis Tsotros, Michael Gounaris, Luis Almeida, Didier Stricker, . . . Nikos Ioannidis. (2001). ARCHEOGUIDE: First results of an Augmented 
Reality, Mobile Com- puting System in Cultural Heritage Sites. doi:10.1145/584993.585015

[12] Qing Hong Gao, Tao Ruan Wan, Wen Tang, Long Chen. (2017). A Stable and Accurate Marker-less Augmented Reality Registration Method. International Conference on Cyberworlds, (pp. 41-47).

[13] Ebrahim Karami, Siva Prasad, Mohamed Shehata. (2015). Image Match- ing Using SIFT, SURF, BRIEF and ORB: Performance Comparison for Distorted Images. Newfoundland Electrical and Computer Engineering Conference. St. johns, Canada [14] Gerhard Reitmayr, Tobias Langlotz, Daniel Wagner , Alessandro Mul- loni, Gerhard Schall, Dieter Schmals, Qi Pan. (2010, July 7-10 ). Simul- taneous Localization and Mapping for Augmented Reality. International Symposium on Ubiquitous Virtual Reality.

[15] A. F. Seber, G., J. Lee, A. (2003). Linear Regression Analysis. Auckland, New Zealand: WILEY

[16] Kifana, B. D., Abdurohman , M. (2012, APRIL 04 ). Great Circle Distance Method for Improving Operational Control System Based on GPS Tracking System. International Journal on Computer Science and Engineering (IJCSE), 04. 